Article

\title{
Improving Tribological Properties of Stainless Steel Surfaces by Femtosecond Laser Irradiation
}

\author{
Serguei P. Murzin ${ }^{1,2, *}$, Valeriy B. Balyakin ${ }^{1}$, Gerhard Liedl ${ }^{2}\left({ }^{\infty}\right.$, Alexey A. Melnikov ${ }^{1}$ and \\ Roland Fürbacher ${ }^{2}$ \\ 1 Samara National Research University, Moskovskoe Shosse 34, Samara 443086, Russian; \\ 029-029@mail.ru (V.B.B.); melnickov.alex@yandex.ru (A.A.M.) \\ 2 Institute of Production Engineering and Photonic Technologies, TU Wien, Getreidemarkt 9, 1060 Vienna, \\ Austria; gerhard.liedl@tuwien.ac.at (G.L.); roland.fuerbacher@tuwien.ac.at (R.F.) \\ * Correspondence: murzin@ssau.ru
}

Received: 29 May 2020; Accepted: 23 June 2020; Published: 28 June 2020

\begin{abstract}
A possibility of improving tribological properties by femtosecond laser irradiation on an example of a steel surface by creating regularly arranged micro-grooved textures that contain self-organized microstructures and nano laser-induced periodic surface structures (LIPSS) was determined. The friction coefficient of the treated surface of a sample of cold-rolled 1.4301 stainless steel was evaluated using a CSM Instruments SA tribometer according to a Pin-on-disk test scheme at a specific pressure of $1 \mathrm{MPa}$. It was found that the coefficient of friction was reduced by $35 \%$ compared to the initial grinded surface. Such laser treatment can find application for using parts in conditions of dry or boundary friction, mainly in units, in which the use of lubricant is unacceptable or extremely undesirable.
\end{abstract}

Keywords: tribological properties; sliding friction coefficient; stainless steel; treated surface; femtosecond laser irradiation; self-organized microstructures; nano LIPSS

\section{Introduction}

Laser treatment, depending on the applied methods, schemes, and processing modes, has a multifactorial effect on the surface condition of the processed materials which has been the subject of numerous studies. To improve tribological properties of materials, along with laser modification methods [1-4], a surface texturing was effectively applied using various laser treatment schemes $[5,6]$. This method has been successfully applied to structural materials, including metals and alloys as well as ceramics, and provides a decrease in the friction coefficient and an increase in the operational characteristics of machine parts and mechanisms [7-9]. Laser texturing is widely used in a variety of applications for the improvement of tribological properties of surfaces [10]. With the aim of industrial application, the texturing was performed on surfaces of mechanical components, such as piston rings [11,12], thrust and rolling bearings [13-15], injection cams [16], facing seals [17], and cutting tools [18]. It was shown that textured surfaces demonstrated a lowered friction coefficient compared to the untreated analogue under dry friction conditions without lubricant as well as boundary and hydrodynamic lubricating conditions. The use of the laser surface treatment of silicon carbide seal rings to reduce the friction coefficient is known in the practice of producing mechanical gas-dynamic seals [19]. In addition to applications for mechanical components, laser texturing was used to improve tribological properties of bio-implant surfaces, such as dental implants and artificial joints, providing better tribological properties of bio-implant materials $[20,21]$. This process was also used to improve tribological properties of drive tapes for electronic devices and showed progressive results [22]. 
Methods of surface structuring by ultrashort laser pulses are also used to improve tribological properties of materials [23-25]. A treatment by a laser beam with a pulse duration in the femtosecond range was performed, implementing an ablation process of the material with transition to the plasma and the gaseous phase, bypassing the liquid melt. In addition to the synthesis of a low-dimensional periodic structure as a result of self-organization processes initiated by femtosecond laser irradiation [26], a direct microrelief formation by laser treatment was applied [27].

Bonse et al. [28] showed that one of the approaches for an application of femtosecond laser texturing to improve the tribological performance of surfaces can be a creation of ripples or laser-induced periodic surface structures (LIPSS) with spatial periods in the sub-micrometer range, which are formed in a "self-ordered" way during the laser-scan processing of surfaces. In Ref. [29], tribological characteristics of periodic surface structures on metallic materials induced by femtosecond laser irradiation were evaluated, including technologically relevant materials such as titanium-containing materials, stainless steels, and steels. In Ref. [30], an influence on the tribological properties of surfaces of metallic materials by self-ordered nano LIPSS as well as by microstructures was shown that also includes so-called grooves and cones morphologies, which often form in a self-organized way. Schille et al. [31] showed that the high potential of laser textured surface features can be demonstrated for an advanced tribological functionality in a real application.

Wang et al. [32] reported on improving tribological properties of a stainless steel by surface microstructuring with patterning using a femtosecond laser. Regularly arranged micro-grooved textures with a different spacing were produced on stainless steel surfaces by a femtosecond laser. It was shown that the femtosecond laser texturing of the surface is an effective technology to improve tribological properties of steels under dry friction. In addition, as shown by Gachot et al. [33] and Zhang et al. [34], such microreliefs may have properties that are able to retain viscous substances, which, for example, makes it possible to create a system of grooves for lubricant accumulation.

Of interest are studies of the functional response of textured surfaces with various designs, including arrays of nanoripples, micro-irregularities, and grooves [9,30]. The aim of this paper is to determine the possibility of improving tribological properties by femtosecond laser irradiation on an example of a steel surface by creating regularly arranged micro-grooved textures containing self-organized microstructures and nano LIPSS.

\section{Materials and Methods}

In this study, 1.4301 stainless steel samples were used with a thickness of $2 \mathrm{~mm}$ and a grinded surface finish. Steel 1.4301 find application in friction assemblies in chemical equipment when functioning in most medium-aggressive environments of chemical industries, including diluted acids and alkalis and organic acids as well as in most salt solutions of organic and inorganic acids at various temperatures and concentrations. In the presence of aggressive environments, parts made of such steel are used at temperatures of up to $350^{\circ} \mathrm{C}$. The chemical composition of $\mathrm{X} 5 \mathrm{CrNi18}-10-1.4301$ steel is presented in Table 1.

Table 1. Chemical composition of X5CrNi18-10-1.4301 steel, weight \%.

\begin{tabular}{ccccccccc}
\hline $\mathbf{N}$ & $\mathbf{N i}$ & $\mathbf{C r}$ & $\mathbf{S}$ & $\mathbf{P}$ & $\mathbf{M n}$ & $\mathbf{S i}$ & $\mathbf{C}$ & $\mathbf{F e}$ \\
\hline$<0.11$ & $8.0-10.5$ & $17.0-19.5$ & $<0.015$ & $<0.045$ & $<2.0$ & $<1.0$ & $<0.07$ & basis \\
\hline
\end{tabular}

The profile measurements of untreated samples showed an arithmetical mean deviation of $\mathrm{Ra}=1.3 \mu \mathrm{m}$ and a maximum height of $\mathrm{Sz}=9 \mu \mathrm{m}$ (according to DIN EN ISO 4287:2010). The surface properties were defined using a roughness measuring instrument, Mahr Marsurf PS 10 (Mahr GmbH, Göttingen, Germany). A Ti:Sapphire ( $\mathrm{Ti}^{\mathrm{A}} \mathrm{Al}_{2} \mathrm{O}_{3}$ ) solid state laser (Femtopower Compact Pro, Femtolasers Produktions $\mathrm{GmbH}$, Vienna, Austria) was used to initiate the LIPSS formation and material removal. The system operates at a frequency of $1 \mathrm{kHz}$, emitting $30 \mathrm{fs}$ pulses at a central wavelength of $\lambda=800 \mathrm{~nm}$ 
and a bandwidth of $\pm 50 \mathrm{~nm}$. The laser delivers radiation with an average output power of $800 \mathrm{~mW}$, which leads to a single pulse energy of $0.8 \mathrm{~mJ}$. Due to its internal setup, the output radiation is linearly polarized. To adjust the laser fluence on the samples' surface, a variable attenuator was used. Leaving the attenuator, the laser beam is then focused on the specimen by an off-axis parabolic mirror with a protected silver coating and a focal length of $50.8 \mathrm{~mm}$ (angle of incidence $0^{\circ}$ ). The processing has been conducted in air at $21^{\circ} \mathrm{C}$. The sample surfaces were cleaned in an ultrasonic bath with demineralized water for 20 minutes after structuring and then examined by a scanning electron microscope (SEM), VEGA TESCAN (Brno, Czech Republic).

Changes in the friction coefficients of treated samples were evaluated using a CSM Instruments SA tribometer (Peseux, Switzerland, Figure 1). The device, designed to measure the coefficient of sliding friction during the contact interaction of two mutually moving surfaces, is a desktop installation consisting of a friction machine with a measuring device and a computer. The method is based on the measurement by a strain gauge of the friction force that occurs from the mutual movement of the test samples pressed against each other with a predefined force in air or in the environment of various lubricants, both consistent and liquid. A Pin-on-disk test scheme was used, in which a flat surface of a fixed cylindrical pin is pressed against a flat sample surface [35]. The friction coefficient is equal to the ratio of the measured friction force to the clamping force.

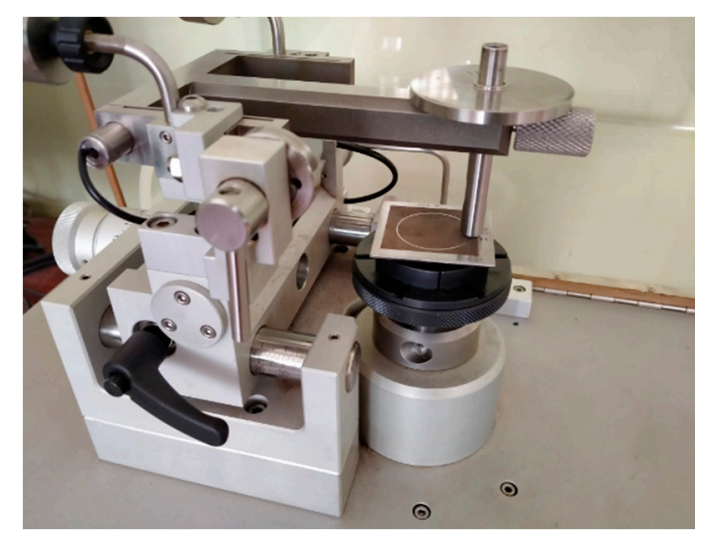

(a)

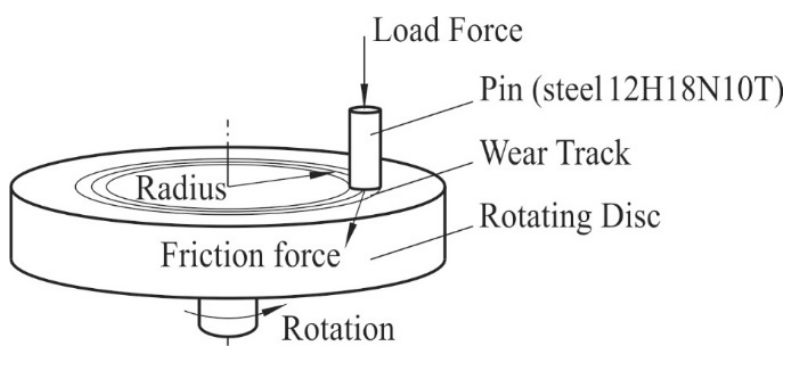

(b)

Figure 1. Determination of friction coefficient: (a) photo of CSM Instruments SA tribometer with a test sample and (b) the test scheme.

In the measurement mode, the samples from cold-rolled 1.4301 stainless steel with an applied micro- and/or nanorelief were sequentially installed on the platform. A counterbody (indenter) made of 12H18N10T steel (an analogue of X10CrNiTi18-10 steel) in the form of a pin fixed motionless in the rod holder was mounted on top of the sample at a predefined distance from the rotation axis of the platform. Before each measurement, a balancing of the holder and the counterbody was performed.

\section{Results and Discussions}

\subsection{Femtosecond Laser Treatment}

The samples of steel were mounted on a positioning system that allows a well-defined translational movement in the $x, y$, and $z$ directions. The movement in the $z$-direction was used to modify the distance of the sample surface to the parabolic focusing mirror. The fluence was adjusted by means of a variable attenuator. The samples' movements along the $x$-axis and stepwise movements between the scanning lines along the $y$-axis were used to form LIPSS or cone-shaped microstructures. The distances between the scanning lines were chosen to allow the overlapping of the formable LIPSS on the 162-I sample surface as well as the periodic cone-shaped microstructures on the 164-I sample surface. This was made to implement a comparison of the tribological properties of nanostructures (LIPSS) that 
were created near the ablation threshold with properties of cone-shaped microstructures. Since LIPSS can only be generated below the ablation threshold of the selected material, the fluence was adjusted by moving the sample out of focus along the z-axis.

Treatment in the $x$-direction followed by another in the $y$-direction were used to generate a grid-shaped microstructure for the 163-I sample. Regularly arranged micro-grooved textures containing self-organized hierarchical structures micro/LIPSS were produced with different distances between the grooves. The gridwise pattern and distances were chosen on the condition that grooves with self-organized hierarchical micro/LIPSS structures will occupy at least 2/3 of the sample area. At the same time, an equal area ratio of more than $10 \%$ for the raw grinded initial surface and formed nano LIPSS was maintained. The used sets of parameters are summarized in Table 2.

Table 2. Sets of parameters for the laser structuring of 1.4301 stainless steel.

\begin{tabular}{ccccc}
\hline Sample ID & Structure Description & Fluence $\boldsymbol{\varphi}\left[\mathbf{J} / \mathbf{c m}^{\mathbf{2}}\right]$ & Feed Rate $\mathbf{f}[\mathbf{m m} / \mathbf{s}]$ & Gap Width a $[\boldsymbol{\mu m}]$ \\
\hline 162-I & Nano, line & 0.44 & 8.33 & 160 \\
$163-\mathrm{I}$ & Micro, grid & 2.74 & 1.67 & 100 \\
$164-\mathrm{I}$ & Micro, grid & 2.74 & 0.42 & 50 \\
\hline
\end{tabular}

Accordingly, the samples were prepared in such a way that different ablation modes were established. For fluences below the ablation threshold, only nanosized LIPSS have been generated. The second sample has been prepared at higher fluences, which caused the formation of a certain microstructure, together with nanosized LIPSS. The third sample was prepared with different parameters, where no LIPSS at all were formed. As a consequence, only microstructures and no LIPSS were present in that sample.

The SEM images of 162-I sample were analyzed perpendicular to the LIPSS orientation using Fast Fourier Transformation (FFT), a fast Fourier transform is an algorithm that computes the discrete Fourier transform, with the MATLab software (version R2018b) to determine the LIPSS periodicity. Therefore, each pixel row of the image was transformed into a frequency domain, and an average frequency distribution over all the rows was calculated. The resulting spectrum was further investigated to reveal distinct peaks. Those peaks can be interpreted as the favorable spatial LIPSS periods, and the amplitude gives an indication of their characteristics. The FFT analysis of the 162-I sample showed a frequency peak at $1.71 \mu^{-1}$, which corresponds to the main spatial period of $0.58 \mu \mathrm{m}$. A local transition to higher spatial frequencies, which was also identified by FFT analysis, can be seen in Figure 2. These local LIPSS frequency transitions are caused by laser intensity variations due to the sample's surface roughness, and this led to local spatial periods of $0.36 \mu \mathrm{m}$.

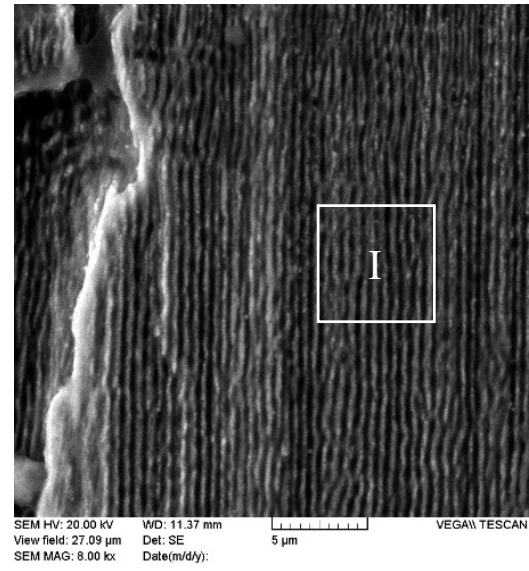

(a)

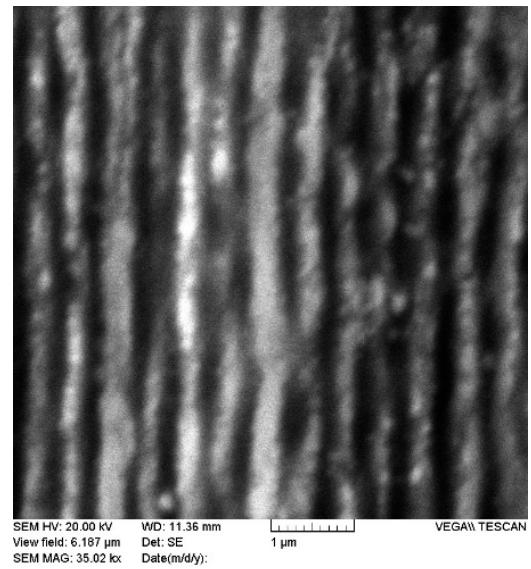

(b)

Figure 2. SEM images: (a) surface nano laser-induced periodic surface structures (LIPSS) formed using a femtosecond laser irradiation on a grinded steel surface; (b) enlargement of the marked area I. 
The SEM images of the micro-structured 163-I sample revealed not only regions where the material was removed through ablation caused by a high fluence but also the formation of LIPSS near the fringes of the ablated zones (Figures 3 and 4). These hierarchical structures with micrometer-sized structures at the bottom and nanometer-sized structures on the top can lead to a change in the behavior of the surface. On the 164-I sample surface LIPSS were absent, but periodic cone-shaped microstructures were present (Figure 5).

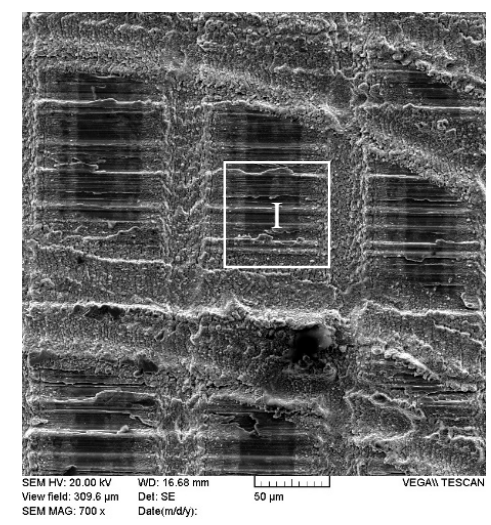

(a)

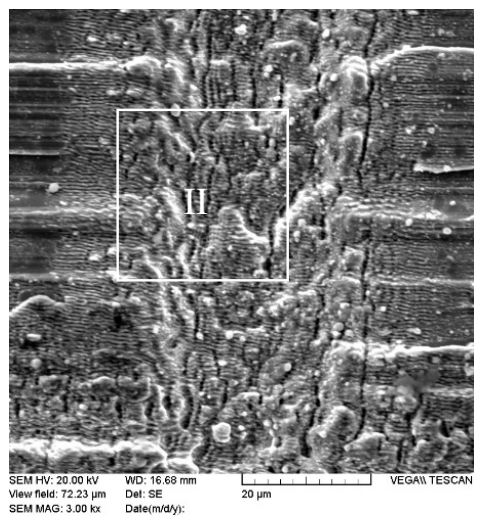

(b)

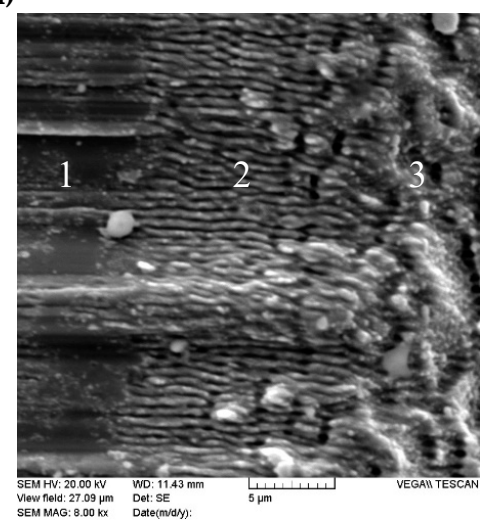

(c)

Figure 3. SEM images: (a) regularly arranged micro-grooved textures containing self-organized hierarchical structures micro/LIPSS; (b) enlargement of the marked area I. (c) Enlargement of the marked area II: 1-initial grinded surface; 2-nano LIPSS; 3-groove with self-organized hierarchical micro/LIPSS structures.

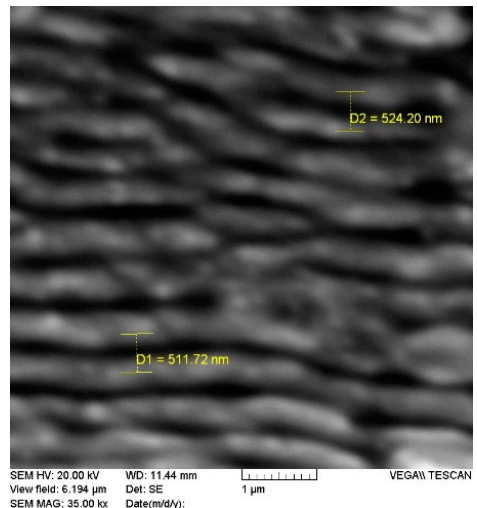

(a)

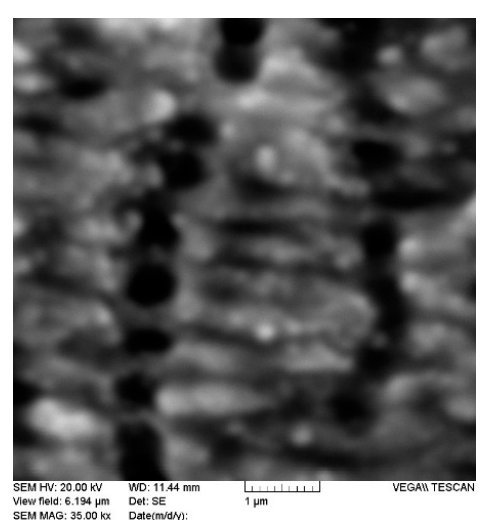

(b)

Figure 4. SEM images: (a) nano LIPSS and (b) self-organized hierarchical structures of micro/LIPSS in the groove. 


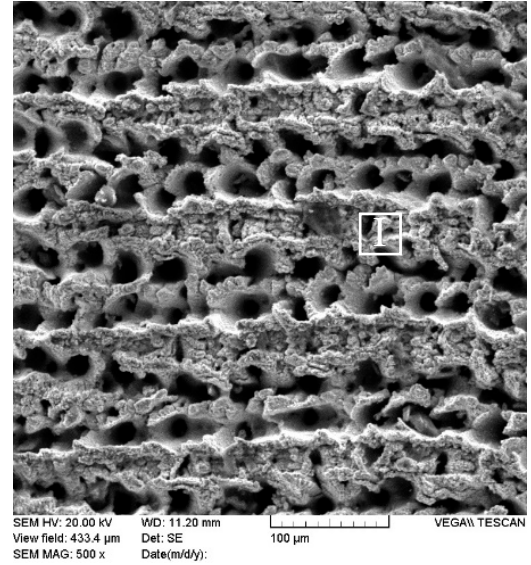

(a)

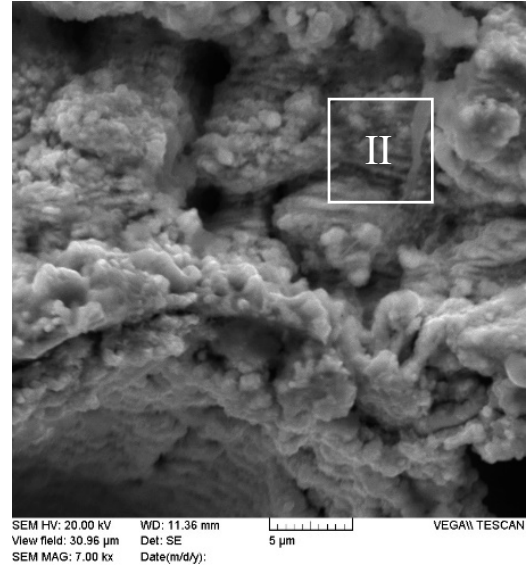

(b)

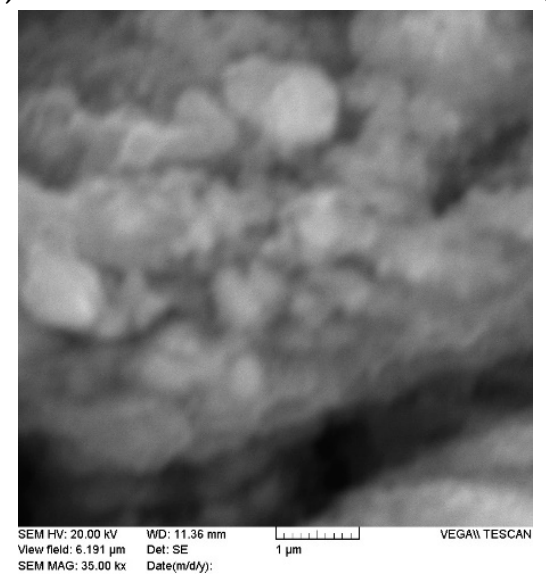

(c)

Figure 5. SEM images: (a) periodic cone-shaped microstructures on the sample surface; (b) enlargement of the marked area I; (c) enlargement of the marked area II.

\subsection{Determination of Sliding Friction Coefficient}

The sliding friction coefficient was determined without lubrication at an ambient temperature of $25^{\circ} \mathrm{C}$ and a relative humidity of $63 \%$. The pressing force was created by placing a weight holder with a known mass on the stand. A specific pressure of $1 \mathrm{MPa}$ was realized at a load force of $1 \mathrm{~N}$, while the linear sliding speed was $0.5 \mathrm{~m} / \mathrm{s}$ and the length of sliding was $50 \mathrm{~m}$, with a test radius of $15 \mathrm{~mm}$. In the device, friction force sensors are connected to the counterbody holder. The friction coefficient was measured during the rotational movement of the sample relative to the counterbody, while the wear groove on the sample had a circle shape. During the tests, the friction coefficient was measured with a frequency of 1000 measurements per minute; these values were statistically processed by the CSM tribometer software package, which determined the maximum, minimum, and average values, as well as the standard deviation. The arithmetic mean values obtained after processing the statistical matrix of more than 1650 measurements for each series of tests were determined. This number of measurements for each series of tests provides a measurement reliability of 0.997 .

The standard deviation of the average value of the friction coefficient was determined by software for experimental equipment for each point and was in the range of $0.002-0.04$. However, a comparison of the friction coefficient before and after the laser surface treatment was performed using an average value. The systematic error was eliminated by calibrating the experimental setup before each series of measurements.

As a result of experimental studies, it was found that for the initial surface of the samples, the average value of the friction coefficient in contact with a specific pressure of $1 \mathrm{MPa}$ was 0.149 , 
with a mean square deviation of 0.002 . Figure 6 shows the dependence of the friction coefficient on the length of sliding for the laser-untreated sample surface. The average value of the friction coefficient for the 162-I sample, on the surface of which a LIPSS nanostructure was formed using femtosecond laser irradiation, was in the range of $0.14-0.15$. Moreover, the maximum value was recorded when the movement started. In the initial 6-7 s, a decrease in the friction coefficient to the minimum value was observed, and afterwards an almost monotonous increase up to the value of 0.145 with a mean square deviation of 0.0025 (Figure 7) was registered.

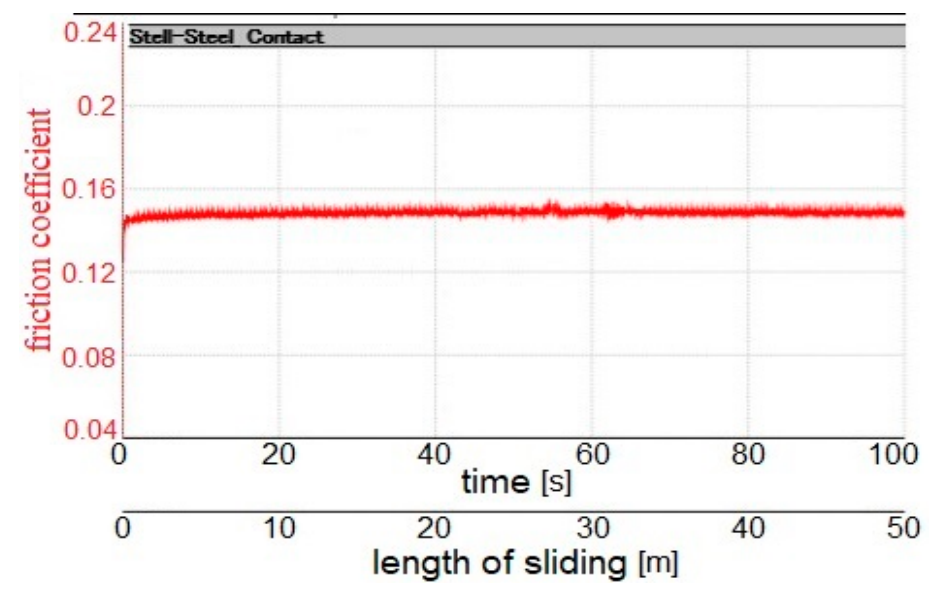

Figure 6. Dependence of the friction coefficient on the length of sliding for the laser-untreated sample surface.

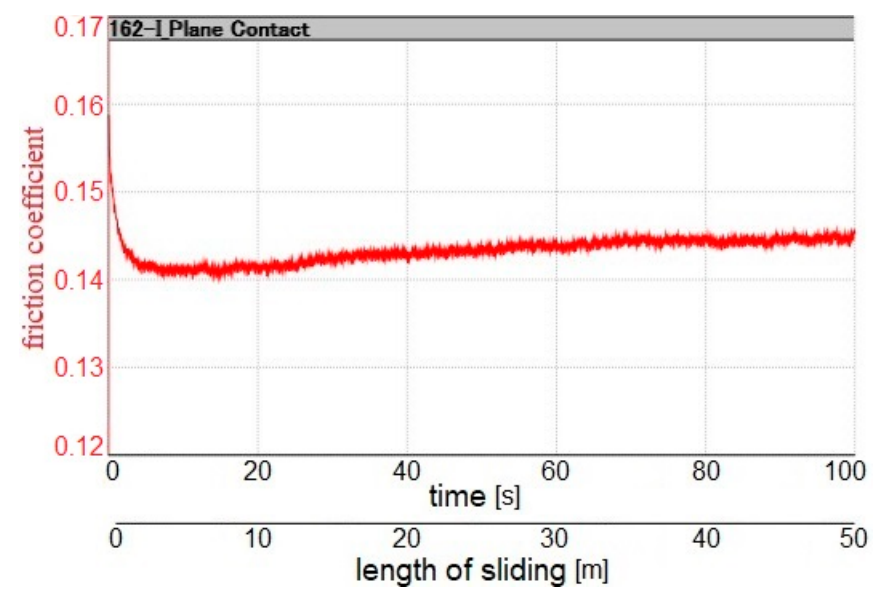

Figure 7. Dependence of the friction coefficient on the length of sliding for the 162-I sample surface.

The friction coefficient for the 163-I sample surface was studied, with its surface showing a hierarchical structure with cones in the micrometer range from the bottom and nanoscaled LIPSS at the top, formed at higher accumulated energy densities. The friction coefficient of the surface, which was a hierarchical structure of micro/LIPSS, was 0.12 at the time when the movement started, and in less than 10 seconds it decreased to 0.1 (Figure 8). Further in the course of the study, a decrease in the coefficient of the sliding friction of the sample to an arithmetic mean value of 0.096 with a mean square deviation of 0.0035 was observed. Thus, it was found that the coefficient of friction was reduced by $35 \%$. For the 164-I sample, the surface of which was characterized by the absence of LIPSS and the presence of larger self-organizing periodic cone-shaped structures that were formed at a further increase in the accumulated energy density after laser treatment, the friction coefficient during the studies increased in the range of 0.1 (at the initial moment) to 0.2 (Figure 9). The average value in this test interval was 0.147 , with a mean square deviation of 0.04 . SEM images of the wear areas of sample surfaces are shown in Figure 10. 


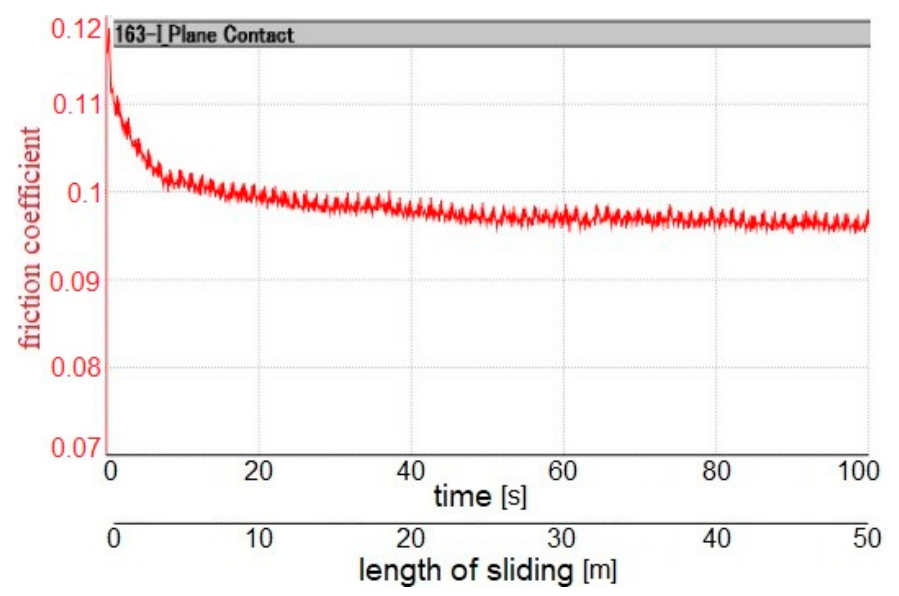

Figure 8. Dependence of the friction coefficient on the length of sliding for the 163-I sample surface.

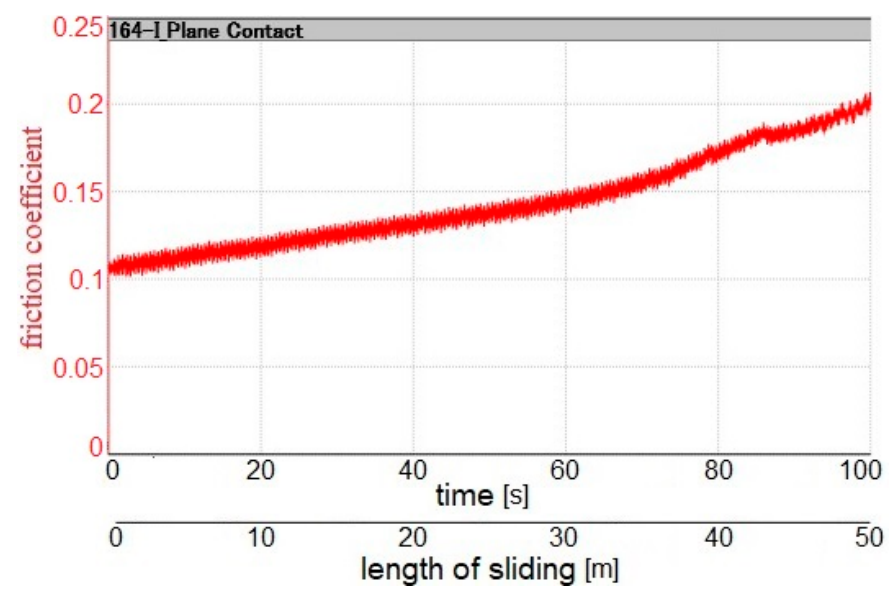

Figure 9. Dependence of the friction coefficient on the length of sliding for the 164-I sample surface.

The change in the friction coefficient during tests is explained by the surface wear of samples. An analysis of the graph from Figure 8 shows that during the tests, the friction coefficient decreased slightly, which is explained by a decrease in the surface roughness due to polishing. A shiny polished surface was observed visually, whose SEM image is shown in Figure 10. Surface wear was measured using an indicator-type micrometer. The wear value of the 163-I sample surface was less than the division interval of the scale, which was $1 \mu \mathrm{m}$. As shown in Figure 7, the friction coefficient of the 162-I sample first decreased and then began to increase slightly, which can be explained by the beginning of the surface wear. The measurement of the depth of the groove showed a wear of $1-2 \mu \mathrm{m}$. The wear during testing of the 164-I sample was 15-17 $\mu \mathrm{m}$, which is by an order greater (Figure 9). In this case, the indenter, which has the shape of a rectangular prism, cut deep into the sample, as a result of which additional resistance appeared and the friction coefficient began to increase linearly. Therefore, it can be claimed that the laser processing of 1.4301 steel by irradiation with a femtosecond laser with the creation of a hierarchical structure with cones in the micrometer range from the bottom side and LIPSS in the nanometer range on the top reduces the friction values for pairs of stainless steel. Thus, the feasibility of creating a hierarchical micro/LIPSS structure to reduce the friction coefficient of steel was determined. 


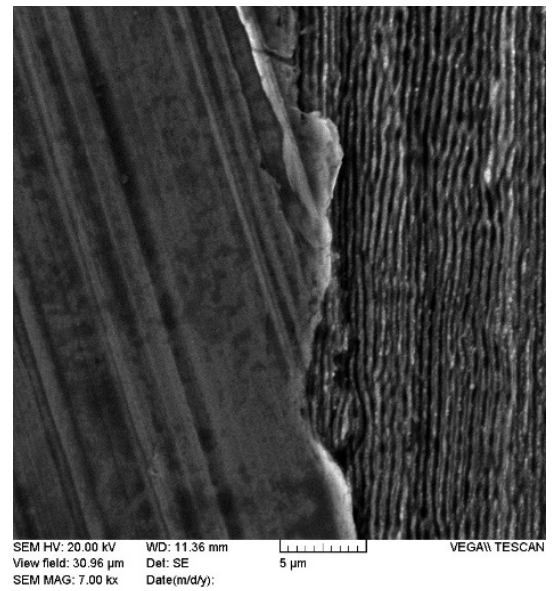

(a)

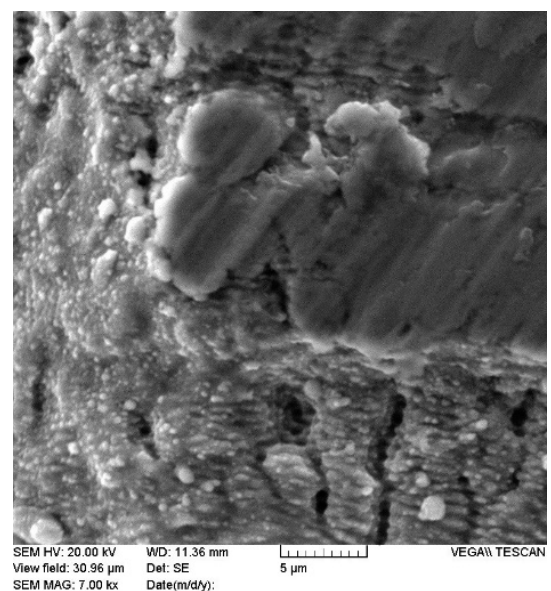

(b)

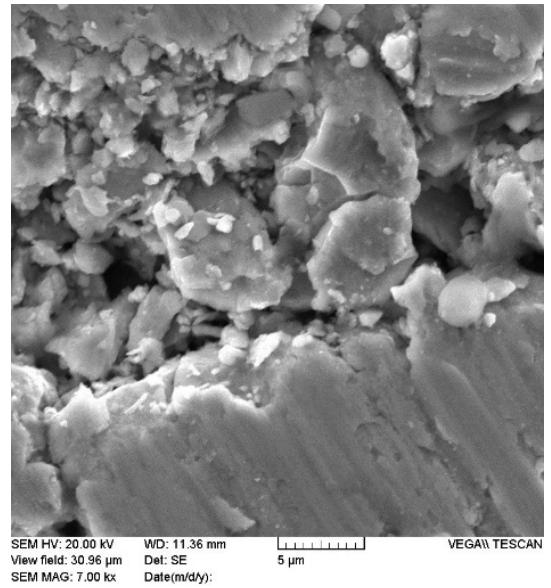

(c)

Figure 10. SEM images of the wear areas of samples surfaces: (a) 162-I, (b) 163-I, (c) and 164-I in the study of the friction coefficient.

\subsection{Application Possibilities in Friction Units}

Such processing can find application when using parts made of 1.4301 steel in friction units of many types of equipment in which the use of lubricants is unacceptable or extremely undesirable. For example, production technology often excludes lubrication in machines used for food, textiles, and paper, as well as chemical industries. In this case, components function in conditions of dry friction, which occurs in the absence of lubrication and contamination between the surfaces. Stainless steel textured surfaces with arrays of nanoripples, micro-irregularities, and grooves can be used in conditions of boundary friction, in which the surfaces are separated by a lubricant layer so thin that it has special properties different from the bulk properties of the lubricant and depending on the nature and condition of the rubbing surfaces.

The main factors that should be taken into account primarily when choosing materials and processing methods are load characteristics (contact pressure, sliding speed), specified technical lifetime (total lifetime of the friction unit), temperature at operating conditions, lubrication conditions (presence and type of lubricant), the kind of environment (atmospheric air or inert gas and their humidity, or vacuum), and requirements for the friction coefficient. Laser processing with surface texturing on a hierarchical scale could prove to be helpful for parts experiencing sliding friction, such as components of centrifuge rotors; sieves; centrifuge screws; mechanical seal ring, e.g., of axial sealing devices; and many more. 


\section{Conclusions}

A possibility of improving tribological properties by femtosecond laser irradiation on the example of a steel surface by creating regular arranged micro-grooved textures containing self-organized microstructures and nano LIPSS was determined. The friction coefficient of the treated surface of samples of cold-rolled 1.4301 stainless steel was studied. This surface had a hierarchical structure with micrometer-sized cones at the bottom and nanoscaled LIPSS at the top. Changes in the friction coefficients of the treated samples were evaluated using a CSM Instruments SA tribometer according to the Pin-on-disk test scheme at a specific pressure of $1 \mathrm{MPa}$.

The measured friction coefficient of the surface, with its regularly arranged micro-grooved texture containing self-organized hierarchical structures of microstructures/LIPSS, was 0.12 at the beginning of the measurement, when the movement started. In less than 10 seconds, the friction coefficient decreased to 0.1. Subsequently, the sliding coefficient of the sample decreased to a value of 0.096 during the study. Thus, it was found that the coefficient of friction was reduced by $35 \%$ compared to the initial grinded surface.

Such processing could be applied to components made of 1.4301 steel used under the conditions of dry or boundary friction, mainly in friction assemblies of machine types in food, textile, paper, and chemical industries, where the use of lubricants is unacceptable or extremely undesirable. Laser treatment with surface texturing can be recommended for parts of sliding friction units-for example, components of centrifuge rotors, sieves, centrifuge screws, mechanical seal rings of axial sealing devices, etc.

Author Contributions: Conceptualization, S.P.M. and G.L.; Methodology, S.P.M., V.B.B., and G.L.; Software, A.A.M. and R.F.; Validation, V.B.B. and G.L.; Formal analysis, G.L.; Investigation, V.B.B., A.A.M., and R.F.; Resources, S.P.M. and G.L.; Data curation, S.P.M., V.B.B. and G.L.; Writing—original draft preparation, S.P.M. and R.F.; Writing-review and editing, S.P.M. and G.L.; Visualization, V.B.B. and A.A.M.; Supervision, S.P.M.; Project administration, S.P.M.; Funding acquisition, S.P.M. and G.L. All authors have read and agreed to the published version of the manuscript.

Funding: This research received no external funding.

Conflicts of Interest: The authors declare no conflict of interest.

\section{References}

1. Wagh, Y.R.; Paul, S.; Gupta, N.; Singh, R.K. Metallurgical and tribological investigation of micro-scale fibre laser-based surface hardening. Int. J. Mechatron. Manuf. Syst. 2018, 11, 120-134.

2. Siddaiah, A.; Mao, B.; Liao, Y.; Menezes, P.L. Surface characterization and tribological performance of laser shock peened steel surfaces. Surf. Coat. Technol. 2018, 351, 188-197. [CrossRef]

3. Sidashov, A.V.; Kozakov, A.T.; Yares'ko, S.I.; Kakovkina, N.G.; Manturov, D.S. Study of the phase composition and tribological properties of carbon tool steels after laser surface hardening by quasi - CW fiber laser. Surf. Coat. Technol. 2020, 385, 125427. [CrossRef]

4. Garcia-Giron, A.; Romano, J.M.; Liang, Y.; Dashtbozorg, B.; Dong, H.; Penchev, P.; Dimov, S.S. Combined surface hardening and laser patterning approach for functionalising stainless steel surfaces. Appl. Surf. Sci. 2018, 439, 516-524. [CrossRef]

5. He, Y.; Zou, P.; Zhu, Z.; Zhu, W.-L.; Yang, X.; Cao, J.; Ehmann, K.F. Design and application of a flexure-based oscillation mechanism for surface texturing. J. Manuf. Process. 2018, 32, 298-306. [CrossRef]

6. Zhan, X.; Yi, P.; Liu, Y.; Xiao, P.; Zhu, X.; Ma, J. Effects of single- and multi-shape laser-textured surfaces on tribological properties under dry friction. Proc. Inst. Mech. Eng. C J. Mech. Eng. Sci. 2020, 234, 1382-1392. [CrossRef]

7. Grützmacher, P.G.; Profito, F.J.; Rosenkranz, A. Multi-scale surface texturing in tribology - current knowledge and future perspectives. Lubricants 2019, 7, 95. [CrossRef]

8. Zhang, W.; Yamashita, S.; Kumazawa, T.; Ozeki, F.; Hyuga, H.; Kita, H. Effect of nanorelief structure formed in situ on tribological properties of ceramics in dry sliding. Ceram. Int. 2019, 45, 13818-13824. [CrossRef]

9. Bhaduri, D.; Batal, A.; Dimov, S.S.; Zhang, Z.; Dong, H.; Fallqvist, M.; M'Saoubi, R. On design and tribological behaviour of laser textured surfaces. Procedia CIRP 2017, 60, 20-25. [CrossRef] 
10. Mao, B.; Siddaiah, A.; Liao, Y.; Menezes, P.L. Laser surface texturing and related techniques for enhancing tribological performance of engineering materials: A review. J. Manuf. Process. 2020, 53, 153-173. [CrossRef]

11. Ryk, G.; Etsion, I. Testing piston rings with partial laser surface texturing for friction reduction. Wear 2006, 261, 792-796. [CrossRef]

12. Ezhilmaran, V.; Vijayaraghavan, L.; Vasa, N. Investigation of $\mathrm{Nd}^{3+}$ : YAG laser aided surface texturing to improve tribological characteristics of piston ring. J. Laser Micro Nanoeng. 2017, 12, 195-202.

13. Etsion, I.; Halperin, G.; Brizmer, V.; Kligerman, Y. Experimental investigation of laser surface textured parallel thrust bearings. Tribol. Lett. 2004, 17, 295-300. [CrossRef]

14. Murzin, S.P.; Balyakin, V.B. Microstructuring the surface of silicon carbide ceramic by laser action for reducing friction losses in rolling bearings. Opt. Laser. Technol. 2017, 88, 96-98. [CrossRef]

15. Houdková, Š.; Šperka, P.; Repka, M.; Martan, J.; Moskal, D. Shifted laser surface texturing for bearings applications. J. Phys. Conf. Ser. 2017, 843, 012076. [CrossRef]

16. Kang, Z.; Fu, Y.; Ji, J.; Puoza, J.C. Effect of local laser surface texturing on tribological performance of injection cam. Int. J. Adv. Manuf. Technol. 2017, 92, 1751-1760. [CrossRef]

17. Wan, Y.; Xiong, D.-S. The effect of laser surface texturing on frictional performance of face seal. J. Mater. Process. Technol. 2008, 197, 96-100. [CrossRef]

18. Xing, Y.; Deng, J.; Zhao, J.; Zhang, G.; Zhang, K. Cutting performance and wear mechanism of nanoscale and microscale textured $\mathrm{Al}_{2} \mathrm{O}_{3} / \mathrm{TiC}$ ceramic tools in dry cutting of hardened steel. Int. J. Refract. Met. Hard. Mater. 2014, 43, 46-58. [CrossRef]

19. Murzin, S.P.; Balyakin, V.B.; Melnikov, A.A.; Vasiliev, N.N.; Lichtner, P.I. Determining ways of improving the tribological properties of the silicon carbide ceramic using a pulse-periodic laser treatment. Comput. Opt. 2015, 39, 64-69. [CrossRef]

20. Hu, T.; Hu, L.; Ding, Q. Effective solution for the tribological problems of Ti-6Al-4V: Combination of laser surface texturing and solid lubricant film. Surf. Coat. Technol. 2012, 206, 5060-5066. [CrossRef]

21. Pfleging, W.; Kumari, R.; Besser, H.; Scharnweber, T.; Majumdar, J.D. Laser surface textured titanium alloy (Ti-6Al-4V): Part 1-surface characterization. Appl. Surf. Sci. 2015, 355, 104-111. [CrossRef]

22. Raeymaekers, B.; Etsion, I.; Talke, F.E. Enhancing tribological performance of the magnetic tape/guide interface by laser surface texturing. Tribol. Lett. 2007, 27, 89-95. [CrossRef]

23. Voyer, J.; Ausserer, F.; Klien, S.; Ristow, A.; Velkavrh, I.; Diem, A.; Zehetner, J.; Stroj, S.; Heidegger, S.; Bertschler, C.; et al. Sub-micro laser modifications of tribological surfaces. Mater. Perform. Charact. 2017, 6, 42-57. [CrossRef]

24. Bathe, R.; Sai Krishna, V.; Nikumb, S.K.; Padmanabham, G. Laser surface texturing of gray cast iron for improving tribological behavior. Appl. Phys. A 2014, 117, 117-123. [CrossRef]

25. Yang, L.; Ding, Y.; Cheng, B.; He, J.; Wang, G.; Wang, Y. Investigations on femtosecond laser modified micro-textured surface with anti-friction property on bearing steel GCr15. Appl. Surf. Sci. 2018, 434, 831-842. [CrossRef]

26. Nayak, B.K.; Gupta, M.C. Self-organized micro/nano structures in metal surfaces by ultrafast laser irradiation. Opt. Lasers Eng. 2010, 48, 940-949. [CrossRef]

27. Lehr, J.; Kietzig, A.-M. Production of homogenous micro-structures by femtosecond laser micro-machining. Opt. Lasers Eng. 2014, 57, 121-129. [CrossRef]

28. Bonse, J.; Koter, R.; Hartelt, M.; Spaltmann, D.; Pentzien, S.; Höhm, S.; Rosenfeld, A.; Krüger, J. Femtosecond laser-induced periodic surface structures on steel and titanium alloy for tribological applications. Appl. Phys. A 2014, 117, 103-110. [CrossRef]

29. Bonse, J.; Koter, R.; Hartelt, M.; Spaltmann, D.; Pentzien, S.; Höhm, S.; Rosenfeld, A.; Krüger, J. Tribological performance of femtosecond laser-induced periodic surface structures on titanium and a high toughness bearing steel. Appl. Surf. Sci. 2015, 336, 21-27. [CrossRef]

30. Bonse, J.; Kirner, S.V.; Griepentrog, M.; Spaltmann, D.; Krüger, J. Femtosecond laser texturing of surfaces for tribological applications. Materials 2018, 11, 801. [CrossRef]

31. Schille, J.; Schneider, L.; Mauersberger, S.; Szokup, S.; Höhn, S.; Pötschke, J.; Reiß, F.; Leidich, E.; Löschner, U. High-Rate laser surface texturing for advanced tribological functionality. Lubricants 2020, 8, 33. [CrossRef]

32. Wang, Z.; Zhao, Q.; Wang, C.; Zhang, Y. Modulation of dry tribological property of stainless steel by femtosecond laser surface texturing. Appl. Phys. A 2015, 119, 1155-1163. [CrossRef] 
33. Gachot, C.; Rosenkranz, A.; Hsu, S.M.; Costa, H.L. A critical assessment of surface texturing for friction and wear improvement. Wear 2017, 372-373, 21-41. [CrossRef]

34. Zhang, J.; Yang, D.; Rosenkranz, A.; Zhang, J.; Zhao, L.; Song, C.; Yan, Y.; Sun, T. Laser surface texturing of stainless steel - effect of pulse duration on texture's morphology and frictional response. Adv. Eng. Mater. 2019, 21, 1801016. [CrossRef]

35. Stachowiak, G.W.; Batchelor, A.W. Experimental Methods in Tribology; Elsevier Science: Amsterdam, The Netherlands, 2004; p. 363.

(C) 2020 by the authors. Licensee MDPI, Basel, Switzerland. This article is an open access article distributed under the terms and conditions of the Creative Commons Attribution (CC BY) license (http://creativecommons.org/licenses/by/4.0/). 